\title{
Finite Thin Cover on an Orthotropic Elastic Half Plane
}

\author{
Federico Oyedeji Falope ${ }^{1}$ and Enrico Radi $^{2}$ \\ ${ }^{1}$ DIEF, Department of Engineering Enzo Ferrari, University of Modena and Reggio Emilia, Via P. Vivarelli 10 Int. 27, \\ 41125 Modena, Italy \\ ${ }^{2}$ DISMI, Department of Sciences and Methods for Engineering, University of Modena and Reggio Emilia, Via Amendola 2, \\ 42122 Reggio Emilia, Italy
}

Correspondence should be addressed to Enrico Radi; eradi@unimore.it

Received 24 December 2015; Accepted 5 October 2016

Academic Editor: Julius Kaplunov

Copyright ( 2016 F. O. Falope and E. Radi. This is an open access article distributed under the Creative Commons Attribution License, which permits unrestricted use, distribution, and reproduction in any medium, provided the original work is properly cited.

The present work deals with the mechanical behaviour of thin films bonded to a homogeneous elastic orthotropic half plane under plain strain condition and infinitesimal strain. Both the film and semi-infinite substrate display linear elastic orthotropic behaviour. By assuming perfect adhesion between film and half plane together with membrane behaviour of the film, the compatibility condition between the coating and substrate leads to a singular integral equation with Cauchy kernel. Such an equation is straightforwardly solved by expanding the unknown interfacial stress in series of Chebyshev polynomials displaying square-root singularity at the film edges. This approach allows handling the singular behaviour of the shear stress and, in turn, reducing the problem to a linear algebraic system of infinite terms. Results are found for two loading cases, with particular reference to concentrated axial forces acting at the edges of the film. The corresponding mode II stress intensity factor has been assessed, thus providing the stress concentrations at both ends of the covering. Possible applications of the results here obtained range from MEMS, NEMS, and solar Silicon cell for energy harvesting to welded joint and building foundation.

\section{Introduction}

A wide class of MEMS and NEMS are based on thin films and coatings technology and the most part of these microdevices involves film and substrate materials which exhibit anisotropic behaviour [1]. As an example, a crystalline undulator (CU) is a special kind of MEMS realized by properly patterning $[2,3]$, through a suitable photolithographic process, a coated ceramic substrate $[4,5]$ which generally consists of a silicon $(\mathrm{Si})$ or germanium $(\mathrm{Ge})$ crystalline plate. As shown in [4], based on the channeling phenomenon, a CU can be used as a compact source of intense and coherent electromagnetic waves, with particular reference to UV and hard $\mathrm{X}$-rays.

Thin films and coatings are also widely used as thermal barriers and protective layers to shield compressors, internal combustion engines, and turbine engine components from gaseous and aqueous aggressive environments in order to prevent excessive wear and oxidation [6].
Thin films and coatings are usually employed for the realization of modern optical devices also, with particular reference to high-reflectance mirrors and lens in the interferometry and spectroscopy fields (optical filters, telescopes, binoculars, etc.).

In the field of civil engineering, sheets or fibre reinforced stiffeners are often modeled as thin membranes bonded to an elastic substrate simulating concrete [7-11] or rigid road pavement [12] (a viscoelastic analysis performed on variable structural systems can be found in $[13,14])$. As a rough approximation, these problems have been modeled by considering beams resting on an elastic support (e.g., [15]).

Thin films and coatings bonded to an elastic substrate generate geometric discontinuities and, in turn, stress concentrations and strain localizations which can drive damaging phenomena like delamination and crack growth. These phenomena can be properly modeled in the framework of both infinitesimal $[16,17]$ and finite elastostatics [18-22]. In order to accurately evaluate the mechanical behaviour of this 


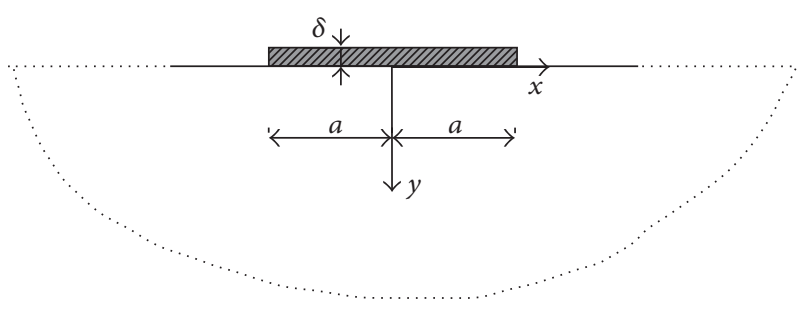

FIGURE 1: Half plane and film's geometry.

kind of micro- or nanosystems, the actual physical properties of the film and substrate materials must be taken into account and the accurate evaluation of the stress and strain fields, particularly along the interface, is mandatory (for nonhomogeneous graded films, nanobeams, and nanoplates, see, e.g., [23-26]).

The mechanical interaction between a thin film and an isotropic half plane has been widely investigated by many researchers through analytical models [5, 27-29] or via numerical analyses [30-32]. The present study investigates the mechanical interaction between a thin elastic film and an elastic orthotropic half plane under various loading conditions. In particular, the present work extends the results obtained in [5] by taking into account anisotropic behaviour of the substrate. The analysis has been performed by assuming plane strain conditions for both the film and the half plane. Moreover, the film is assumed very thin, thus allowing for neglecting its bending stiffness. The strain compatibility condition between the film and the underlying substrate leads to a singular integral equation with Cauchy kernel. By expanding the unknown interfacial shear stress in singular series of Chebyshev polynomials, the integral equation is reduced to an algebraic linear system of infinite terms which is solved for the coefficients of the series expansion. The shear stress intensity factors are then evaluated and compared for two different cases.

The paper is organized as follows: the formulation of the problem in terms of singular integral equation with the Cauchy kernel is presented in Section 2. In Section 3, effective values for material and geometric parameters governing the problem are quantified. The effect induced by the slenderness of the film with respect to the compliance of the half plane is discussed in Section 4 by studying two different loading cases $\gamma$. Finally, conclusions are drawn in Section 5.

\section{Half Plane Covered by Thin Film}

In the present section, the problem of an orthotropic elastic half plane covered by a thin film and subjected to two opposite or concordant axial loads (Figures 1 and 2) is treated. Perfect adhesion between the half plane and the film and negligible bending stiffness of the film have been assumed also. The present analysis could also be extended by assuming a proper constitutive law for the interface in order to simulate imperfect welding, detachment, and so on (e.g., [39]). Under these statements, only the tangential component of the interfacial stress arises within the contact region (Figure 2). The strain fields along the contact region can be written in terms of the interface shear stress $\tau(x)$ by using Green's function for the elastic half plane and by the membrane constitutive equation:

$$
\begin{aligned}
& \varepsilon_{F}=K_{F} \int_{x}^{a} \tau(t) d t-\Delta \varepsilon, \\
& \varepsilon_{S}=\frac{K_{S}}{\pi} \int_{-a}^{a} \frac{\tau(t)}{y-t} d t .
\end{aligned}
$$

Then, the compatibility condition under perfect adhesion assumption leads to the following integral equation:

$$
K_{F} \int_{x}^{a} \tau(t) d t-\Delta \varepsilon=\frac{K_{S}}{\pi} \int_{-a}^{a} \frac{\tau(t)}{x-t} d t,
$$

where $K_{i}$ ( $i=F, S$ stands for "film" or "substrate," resp.; see (A.3)) represents the compliance of the $i$ component. The load term, $\Delta \varepsilon$, has been considered acting on the film only; namely,

$$
\begin{aligned}
& \Delta \varepsilon=-F K_{F} \\
& \Delta \varepsilon=-\left[\left(1+\frac{\alpha_{3 F}}{\alpha_{1 F}} v_{31}\right) \alpha_{S}-\left(1+\frac{\alpha_{3 S}}{\alpha_{1 F}}\right) \alpha_{F}\right] \Delta T,
\end{aligned}
$$

for the case of two opposite axial forces of magnitude $F$ or thermal load variation $\Delta T$, respectively (for a detailed study about the thermoelasticity without energy dissipation, see [40]). By using the Chebyshev polynomials of the first kind, $T_{n}(t)$, the following series expansion has been assumed for the shear stress:

$$
\tau(x)=\frac{\sum_{n=0}^{\infty} C_{n} T_{n}(x / a)}{K_{S} \sqrt{1-(x / a)^{2}}}, \quad \text { for }-a \leq x \leq a .
$$

Introducing the series expansion (4) in (2) using the orthogonality properties of Chebyshev polynomials (Appendix B), multiplying by Chebyshev polynomials of second type $U_{m-1}(t)$ with $m=0,1,2 \ldots$, and integrating over $[-a,+a]$, the integral equation is transformed in a linear algebraic system for the unknown coefficient $C_{n}$. From (2) and (4), it follows that

$$
\begin{aligned}
& \sum_{n=1}^{\infty} C_{n}\left[\frac{\pi \gamma}{2 n} \delta_{n m}+B_{n m}\right] \\
& \quad=\Delta \varepsilon\left\{\frac{1-\cos (m \pi)}{m}+\frac{1-\cos [(m+1) \pi]}{\gamma \pi}\right\} \\
& B_{n m}=\int_{-1}^{+1} U_{n-1}(\xi) U_{m-1}(\xi) d \xi, \quad \text { with } m=1,2 \ldots,
\end{aligned}
$$

where $\gamma=a K_{F} / K_{S}$ is a dimensionless parameter which governs the problem, whose role will be discussed in Section 4, and $\delta_{n m}$ is the Kronecker delta, respectively.

For the loading case sketched in Figure 2(b), the coefficient $C_{0}$ is provided by (4) together with the equilibrium condition of the film:

$$
C_{0}=\frac{\Delta \varepsilon}{\gamma \pi}
$$




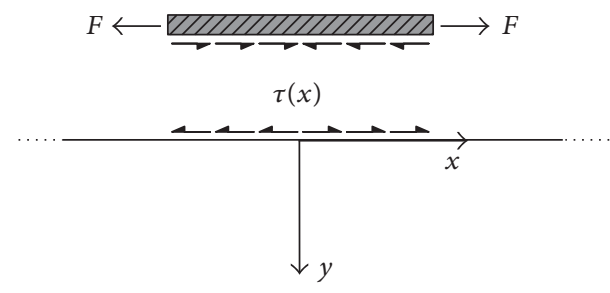

(a) Symmetric loading case

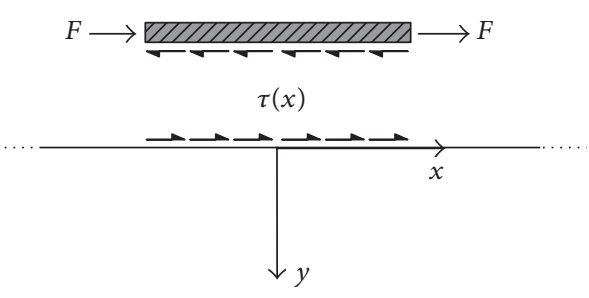

(b) Skew-symmetric loading case

Figure 2: Loading cases.

By introducing the dimensionless variable $\xi=x / a$ over the contact domain, the solution of system (5) with respect to the series coefficient provides

$$
\begin{aligned}
& \varepsilon_{x}^{S}(\xi)=\sum_{n=1,2,3}^{\infty} C_{n} U_{n-1}(\xi), \\
& \varepsilon_{x}^{F}(\xi)=-\Delta \varepsilon+\gamma\left[C_{0} \arccos (\xi)\right. \\
& \left.+\sum_{n=1}^{\infty} C_{n} \frac{U_{n-1}(\xi)}{n} \sqrt{1-\xi^{2}}\right], \\
& \tau(\xi)=\frac{1}{K_{S} \sqrt{1-\xi^{2}}} \sum_{n=0}^{\infty} C_{n} T_{n}(\xi), \\
& \sigma_{x}^{F}(\xi)=\frac{\varepsilon_{y}^{F}(\xi)}{\delta K_{F}}, \\
& u^{F}(\xi)=a\{-\Delta \varepsilon \xi \\
& \left.+\sum_{n=2}^{\infty} \frac{C_{n}}{n} \frac{n T_{n}(\xi)-\xi U_{n-1}(\xi)}{n^{2}-1}\right\}, \\
& \left.+C_{1} \frac{\arccos (\xi)+\xi \sqrt{1-\xi^{2}}}{2}\left(1-\sqrt{1-\xi^{2}}+\xi \arccos (\xi)\right)\right]
\end{aligned}
$$

with $\varepsilon_{x}, \tau, \sigma_{x}$, and $u$ being the longitudinal strain, shear stress, longitudinal stress, and longitudinal displacement, respectively. As a result of the assumption of perfect adhesion between the half plane and the film, expressions (8) and (9) must coincide.

\section{Influence of the Parameter $\gamma$}

The dimensionless parameter $\gamma$ denotes the ratio between the substrate and film compliances. This factor governs the problem and affects the convergence of the Chebyshev series and the stress intensity factor $K_{\mathrm{II}}$ at the edges of the film.

Values of parameter $\gamma$, for different typical materials and film geometries involved in contact problems, can be found in Tables 1 and 2, respectively.
TABLE 1: Some materials involved in contact problems application.

\begin{tabular}{lccc}
\hline Material & Ref & $\delta K_{F}\left[\mathrm{~N}^{-1} \mathrm{~mm}^{2}\right]$ & $K_{S}\left[\mathrm{~N}^{-1} \mathrm{~mm}^{2}\right]$ \\
\hline Seabed soil & {$[33]$} & $9.97 E-1$ & 1.27 \\
Ice & {$[34]$} & $9.76 E-3$ & $9.82 E-3$ \\
Structural steel & {$[35]$} & $4.33 E-5$ & $8.67 E-5$ \\
Beech & {$[36]$} & $4.32 E-5$ & $8.44 E-5$ \\
Silicon nitride & {$[37]$} & $3.23 E-5$ & $6.47 E-5$ \\
Aluminium & {$[34]$} & $1.22 E-5$ & $2.44 E-5$ \\
Silicon & {$[38]$} & $5.15 E-6$ & $1.21 E-5$ \\
Spruce & {$[36]$} & $1.45 E-6$ & $4.88 E-6$ \\
Germanium & {$[34]$} & $8.89 E-7$ & $3.84 E-6$ \\
\hline
\end{tabular}

TABLE 2: Range of film length ratio in contact problems applications.

\begin{tabular}{lcccc}
\hline & $\begin{array}{c}\text { Welded or } \\
\text { bolted } \\
\text { plates }\end{array}$ & $\begin{array}{c}\mathrm{Cu} \text { and } \\
\text { silicon } \\
\text { solar cells }\end{array}$ & Ice lenses & $\begin{array}{c}\text { Slab } \\
\text { foundations }\end{array}$ \\
\hline $\bar{a}$ & $10^{1}-10^{2}$ & $10^{3}-10^{4}$ & $10^{2}-10^{3}$ & $10^{2}-10^{3}$ \\
\hline
\end{tabular}

The limit case occurring for high values of $\gamma$, ranging within $\left[10^{-1}, 10^{2}\right]$, is representative of contact problems met in MEMS and NEMS application. In this case, the half plane is stiffer than the film.

The other limit case concerns low values of $\gamma$, ranging within $\left[10^{-3}, 10^{-6}\right]$, occurring when the stiffness of the half plane is more compliant than the film or when the film has a squat geometry, typically involved in soil contact application.

\section{Results and Discussion}

Two different loading conditions, sketched in Figure 2, have been analysed. The Chebyshev coefficient index has been assumed odd or even including $C_{0}$, for symmetric and skew-symmetric loading cases, respectively. The proper superposition of the two schemes provides the same results reported in [5], where an isotropic film subjected to a single axial force applied to a film end has been studied.

As reported in $[27,28,41]$, both the high speed convergence of the coefficients $C_{n}$ and the regularity of system (5) depend on the parameter $\gamma$. By truncating the Chebyshev series to $n=40$ (20 terms in series expansion), Figure 3 and Table 3 show the convergence of the Chebyshev coefficients. 


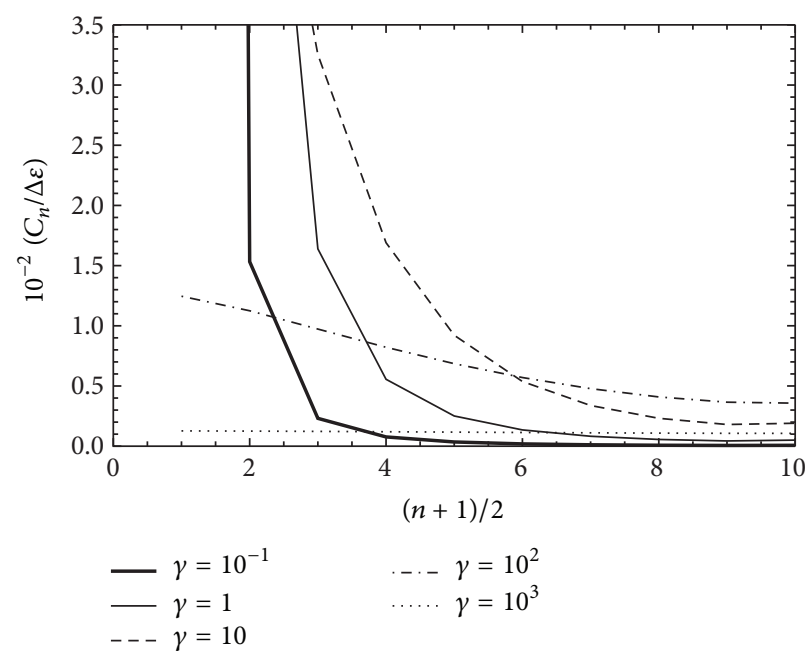

(a) Symmetric loading case, high $\gamma$ values

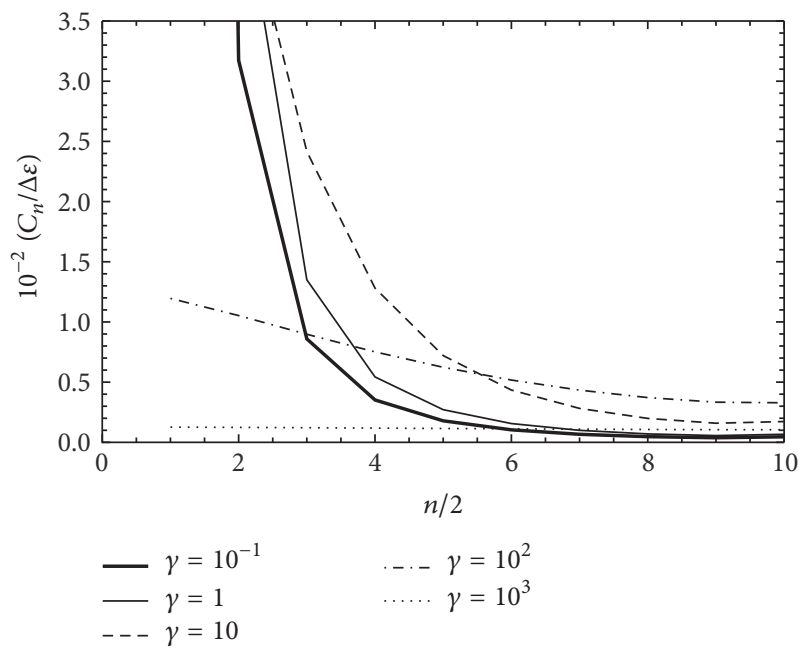

(c) Skew-symmetric loading case, high $\gamma$ values

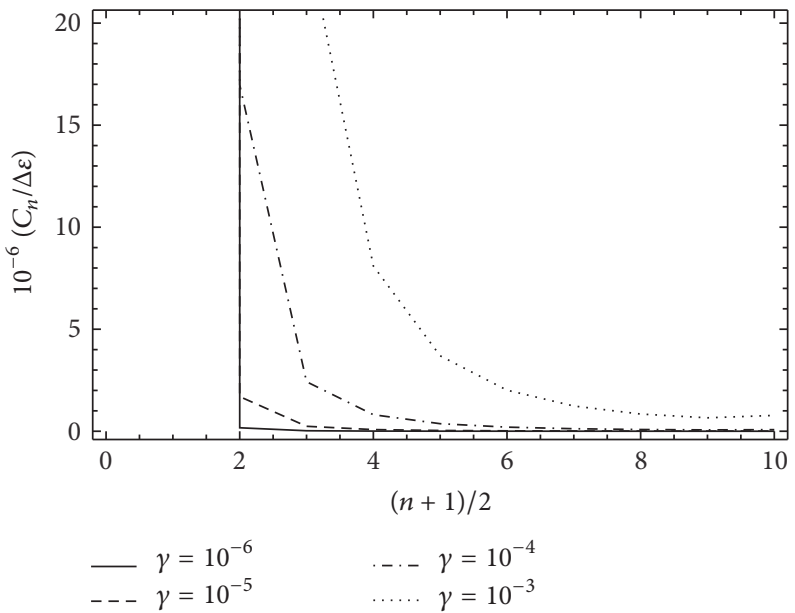

(b) Symmetric loading case, low $\gamma$ values

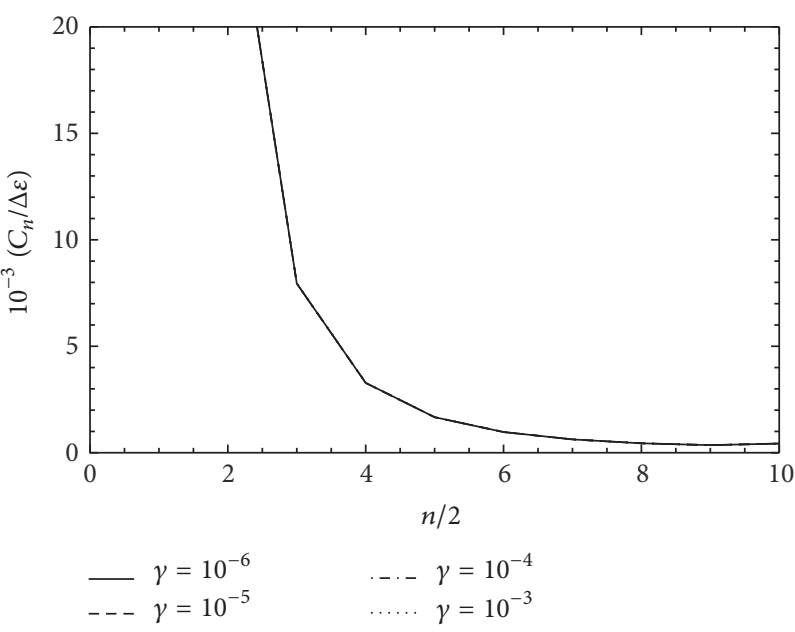

(d) Skew-symmetric loading case, low $\gamma$ values

Figure 3: Convergence of Chebyshev coefficients.

TABLE 3: Sensibility of $C_{n}$ with respect to $\gamma$.

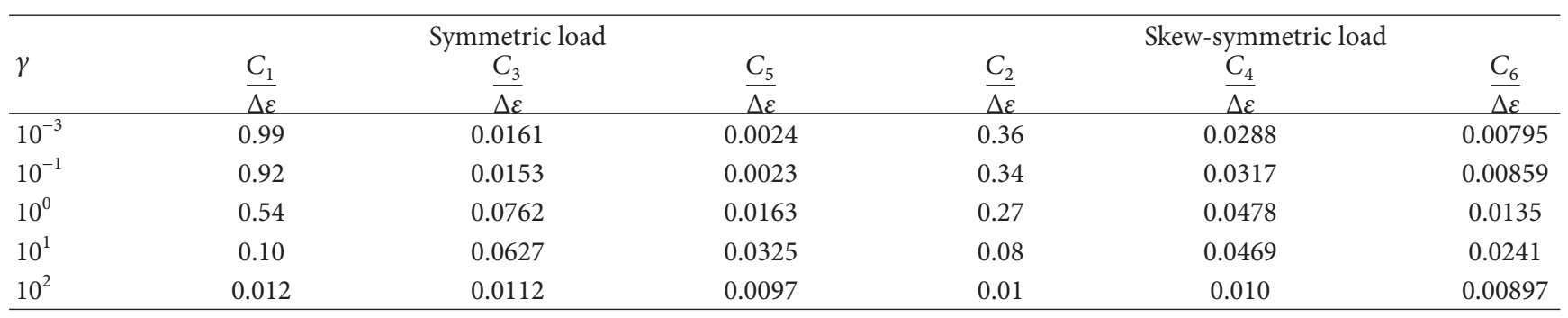

The convergence rate of the series decreases as $\gamma$ increases. For $\gamma>10$, the rate of convergence for the coefficients $C_{n}$ becomes faster. The symmetric loading case exhibits higher convergence speed than the skew-symmetric case.

Under symmetric loading (Figure 2(a)), the longitudinal strain of the substrate (Figure 4) exhibits high sensitivity with respect to the governing parameter only for large value $\gamma$; instead, for small value of $\gamma$, no significant impact on the strain behaviour is detected. For $\gamma<10^{-4}$, the film behaves like a rigid body. Similar to the strain field, the longitudinal displacement increases as the film's compliance increases with $\gamma$ (Figure 5). For low values of $\gamma$, the film displacement varies linearly and attains a maximum value $a \Delta \varepsilon$ at the film edges. For large values of $\gamma$, the axial stress of the film displays an oscillatory behaviour induced by the low convergence rate of the Chebyshev coefficient and by the high gradient of stress 

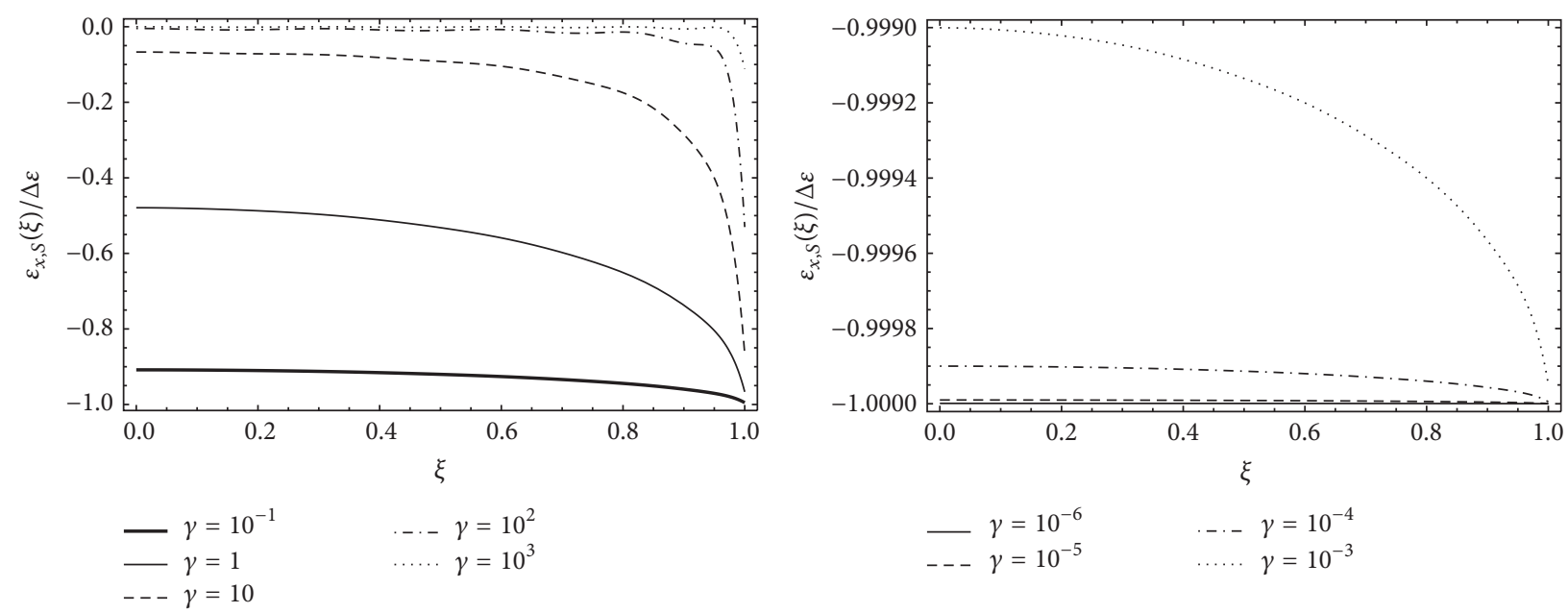

Figure 4: Longitudinal strain field for symmetric loading case.

field in the neighbourhood of the film's edges (Figure 5). As $\gamma$ increases, the magnitude of shear stress field increases and its gradient decreases at the film edge (Figure 2(a)). This behaviour is explained by the stress intensity factor $K_{\mathrm{II}}$ at the films edge which monotonically decreases as $\gamma$ increases (Figure 6).

For the symmetric loading case, the stress intensity factor is defined as proposed by [5]:

$$
\begin{aligned}
K_{\mathrm{II}}( \pm 1) & =\lim _{\xi \rightarrow \pm 1} \tau(\xi) \sqrt{2 \pi(\xi \mp 1)} \\
& =\sqrt{\pi} \sum_{n=1,3, \ldots}^{\infty} \frac{C_{n} T_{n}(\xi)}{K_{S}} .
\end{aligned}
$$

In agreement with the asymptotic solution for isotropic materials in [28], for inextensible films, one finds

$$
\lim _{\gamma \rightarrow 0} \sum_{n=1,3 \ldots}^{\infty} \frac{C_{n} T_{n}}{\Delta \varepsilon}=\frac{C_{1}}{\Delta \varepsilon}=1,
$$

and the stress intensity factor then becomes

$$
K_{\mathrm{II}}(\xi= \pm 1)= \pm \sqrt{\pi} \frac{\Delta \varepsilon}{K_{S}}
$$

in agreement with the value found in $[5,42]$ for isotopic constitutive law. By scaling, the term $\sqrt{2 \pi}$ from the definition of the stress intensity factor $(13)$ provides $K_{\mathrm{II}}(\xi= \pm 1)=$ 0.707 , corresponding to the mode II stress intensity factor at crack tip, induced by the stress component $\tau(\xi= \pm 1)$ which may cause crack propagation along the $\pm \pi / 4$ direction.

The same conclusion drawn about the stress and displacement fields under symmetric loading conditions could be extended to skew-symmetric loading condition (Figures 7 and 8) with the exception of the convergence rate and the stress intensity factor witch are less sensible to the parameter $\gamma$.

Differently from the symmetric loading case, the singularity of the shear stress field is proportional by the Chebyshev constant $C_{0}$ (7). The mode II of the stress intensity factor of the stress intensity factor assumes the following form:

$$
\begin{aligned}
K_{\mathrm{II}}(\xi= \pm 1) & =\lim _{\xi \rightarrow \pm 1} \tau(\xi) \frac{\sqrt{2 \pi(\xi \mp 1)}}{C_{0}} \\
& =\sqrt{\pi} \frac{\Delta \varepsilon}{K_{S}}\left(1+\sum_{n=0}^{\infty} \frac{C_{n} T_{m}(\xi)}{C_{0}}\right) .
\end{aligned}
$$

In the case of an inextensible film, namely, as $\gamma \rightarrow 0$, the sum in (16) becomes vanishing small. As a consequence for this asymptotic case, the stress intensity factor displays the same behaviour of the symmetric loading case (13) as reported in Figure 6.

\section{Conclusions}

The contact problem of a homogeneous orthotropic half plane covered by thin film has been analysed here (for the behaviours of nonhomogeneous nanobeams and nanoplates, see [43-45]). The interfacial singular shear stress has been approximated through a series expansion of Chebyshev polynomials. The governing singular integral equation derived from the compatibility condition between the half plane and thin film has been transformed into a linear algebraic system for the unknown coefficient $C_{n}$ of the series. Once the Chebyshev coefficients have been calculated, the strain field, contact shear stress, and displacement field have been found in dimensionless form in terms of Chebyshev polynomials of the first and second kind.

The governing parameter $\gamma$ assumes specific values for real applications. The rate of convergence of the proposed method displays high or low speed for low $\left[10^{-6}, 10^{-3}\right]$ or high $\left[10^{-1}, 10^{3}\right]$ values of $\gamma$, respectively. The relation between the parameter $\gamma$ and the field of practical application (Table 2) suggests the appropriateness of the proposed method for geometries and material properties typically involved in MEMS or steel joints design. 


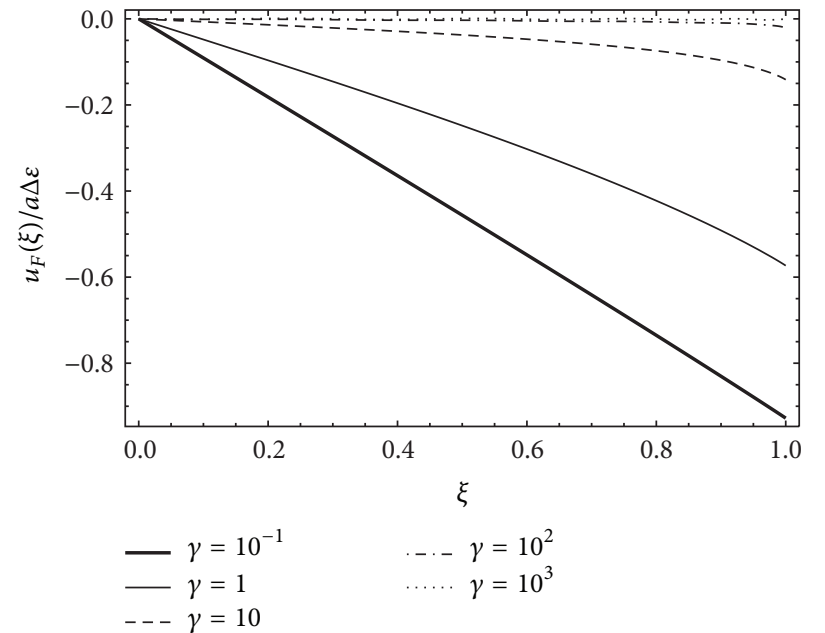

(a)

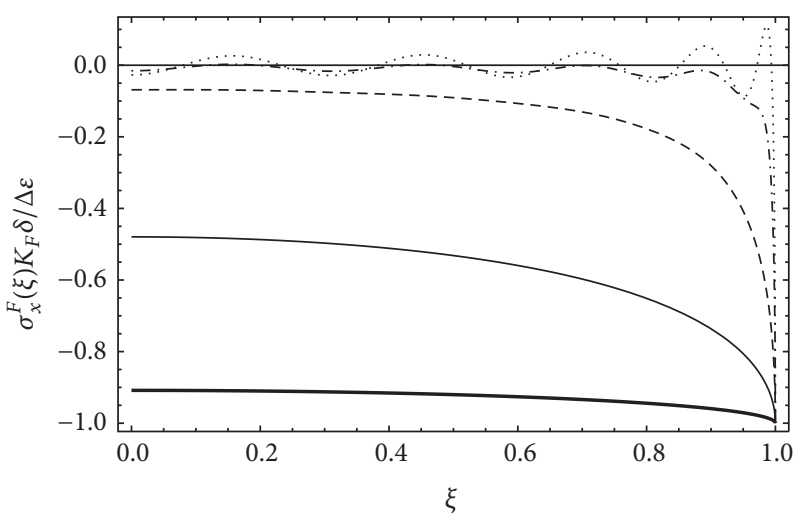

$$
\begin{aligned}
-\gamma & =10^{-1} \\
-\gamma & =1 \\
--\gamma & =10
\end{aligned}
$$

(c)

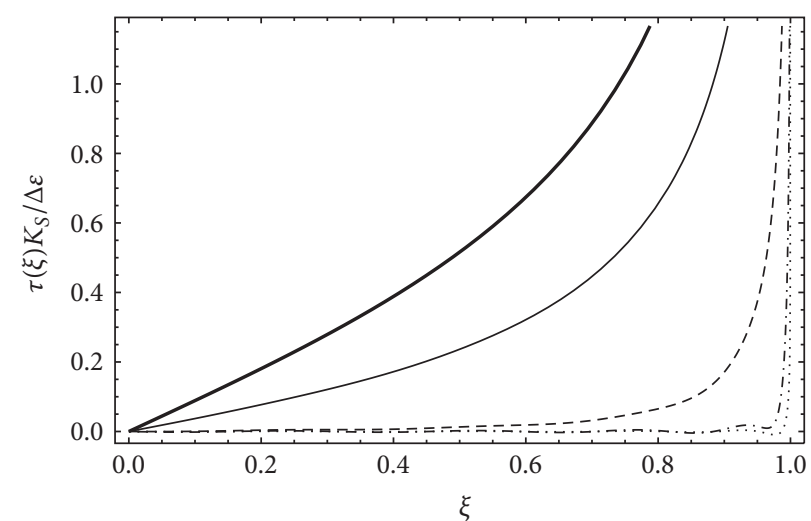

$$
\begin{array}{rlrl}
\gamma & =10^{-1} & \ldots-\gamma=10^{2} \\
-\gamma & =1 & \ldots . & \gamma=10^{3} \\
--\gamma & =10 & &
\end{array}
$$

(e)

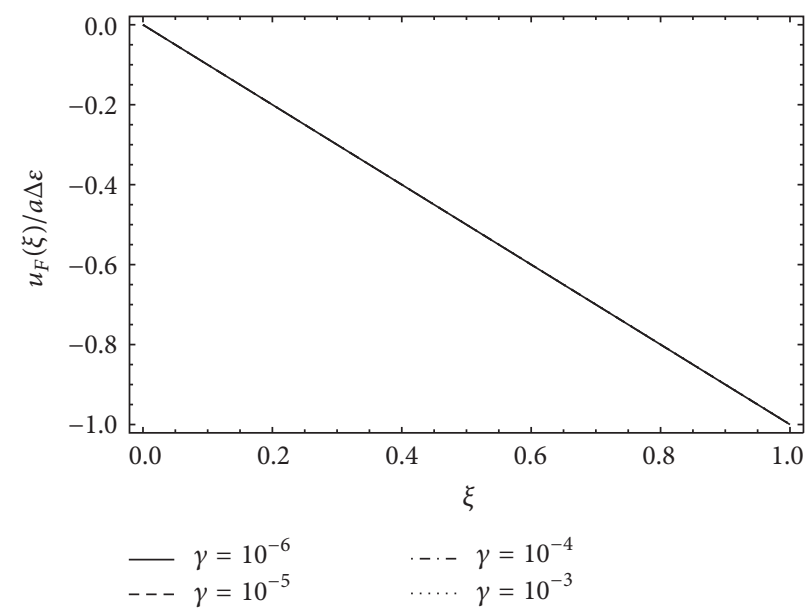

(b)

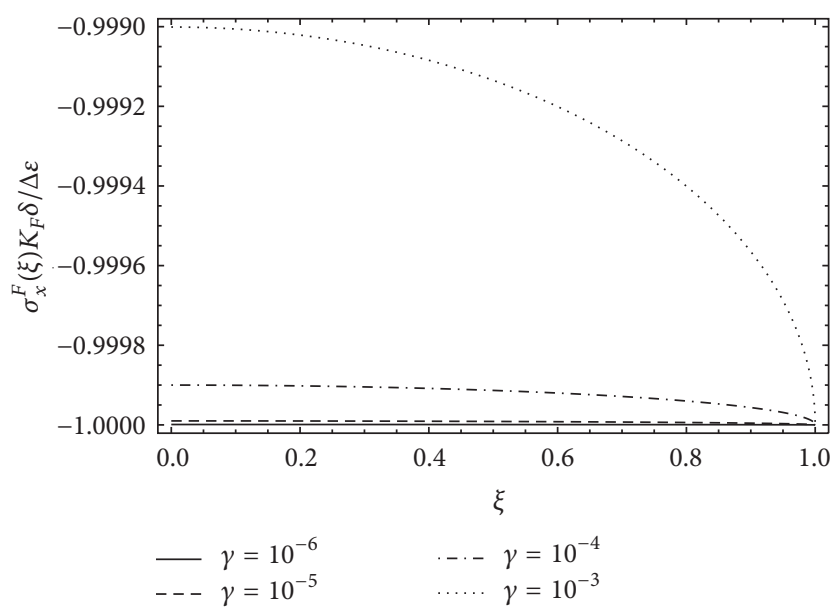

(d)

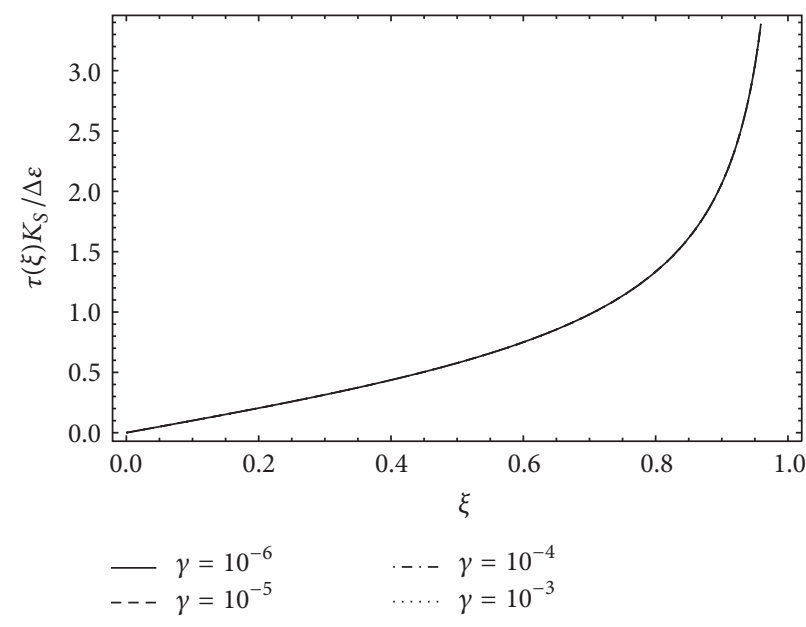

(f)

FIGURE 5: Longitudinal displacement, longitudinal stress, and shear stress field of symmetric loading case condition for high (left (a), (c), and (e)) and low (right (b), (d), and (f)) values of $\gamma$, respectively. 


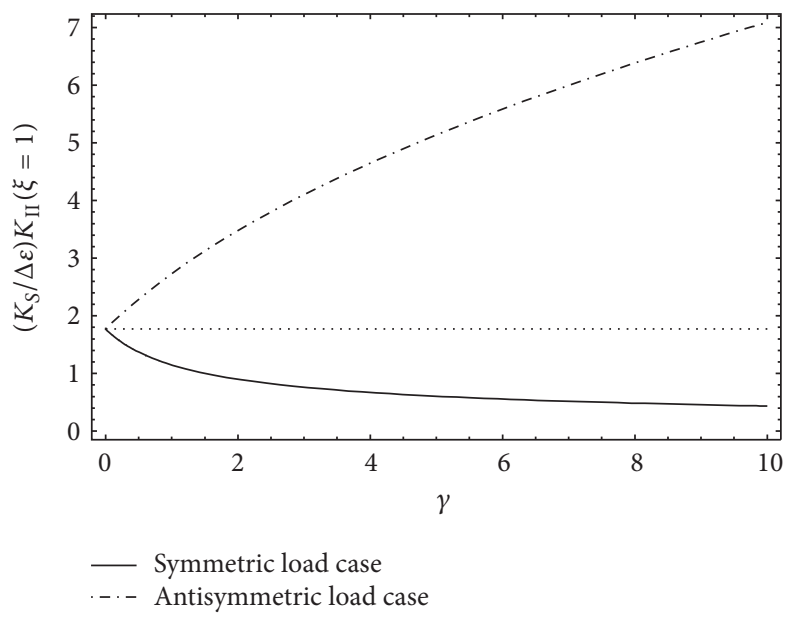

Figure 6: Stress intensity factor at film edge for symmetric loading case.
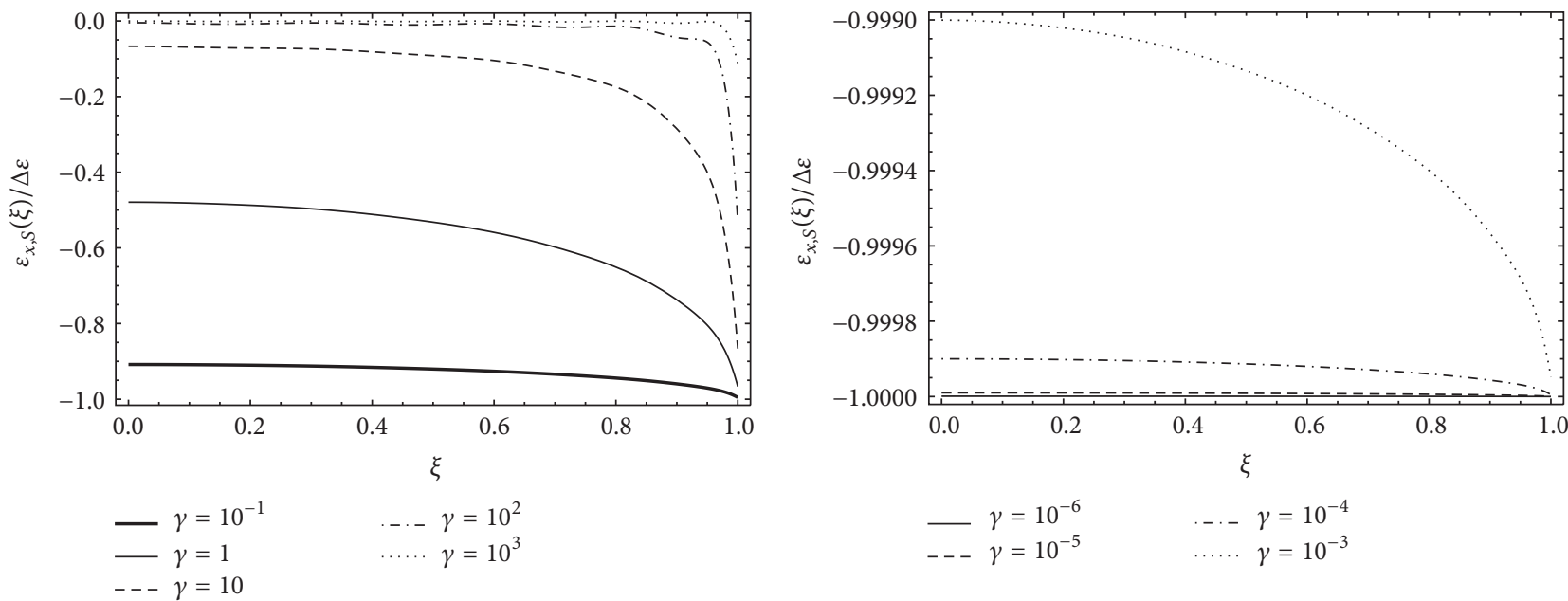

FIGURE 7: Longitudinal strain field for symmetric loading case.

Through the superposition of the two loading conditions here studied, it becomes possible to reproduce the case of a single force acting at the film edge. The stress intensity factors for both symmetric and skew-symmetric loading conditions exhibit opposite behaviour with respect to the dimensionless parameter $\gamma$. The results found here agree reasonably well with the solution reported in $[5,42]$ for the particular case of an isotropic half plane.

In order to take into account voids, defects, and inclusions that can occur during the deposition process of a film onto a substrate, the analysis will be extended to a nonhomogeneous half plane in a forthcoming paper (for a detailed study concerning nonhomogeneous bodies, see [46]).

\section{Appendix}

\section{A. Elastic Parameters}

Denoting with $c_{i j}$ the elastic stiffness coefficients, with $E$ and $v$ being the Young modulus and the Poisson ratio, respectively, from [16], the horizontal component of strain of the half plane surface is known in closed form:

$$
\varepsilon^{S}(y)=\frac{\beta_{22} \sqrt{C_{h}+4}}{\lambda \pi} \int_{-a}^{+a} \frac{\tau(t)}{y-t} d t,
$$

where the elastic parameters read

$$
\begin{aligned}
\beta_{22} & =\frac{c_{11}}{c_{11} c_{22}-c_{12}^{2}}, \\
\lambda & =\sqrt[4]{\frac{c_{11}}{c_{22}},} \\
C_{h} & =\frac{\left(\widetilde{c}_{12}+c_{12}\right)\left(\widetilde{c}_{12}-c_{12}-2 c_{66}\right)}{\widetilde{c}_{12} c_{66}}, \\
R & =\frac{\left(c_{12}-\lambda^{2} c_{22}\right)\left(c_{12}+c_{66}\right)}{\left[\lambda^{4} c_{22}^{2}+\lambda^{2} c_{12} c_{22}\left(C_{h}+2\right)+c_{12}^{2}\right] c_{66}}, \\
\tilde{c}_{12} & =\sqrt{c_{11} c_{22}},
\end{aligned}
$$




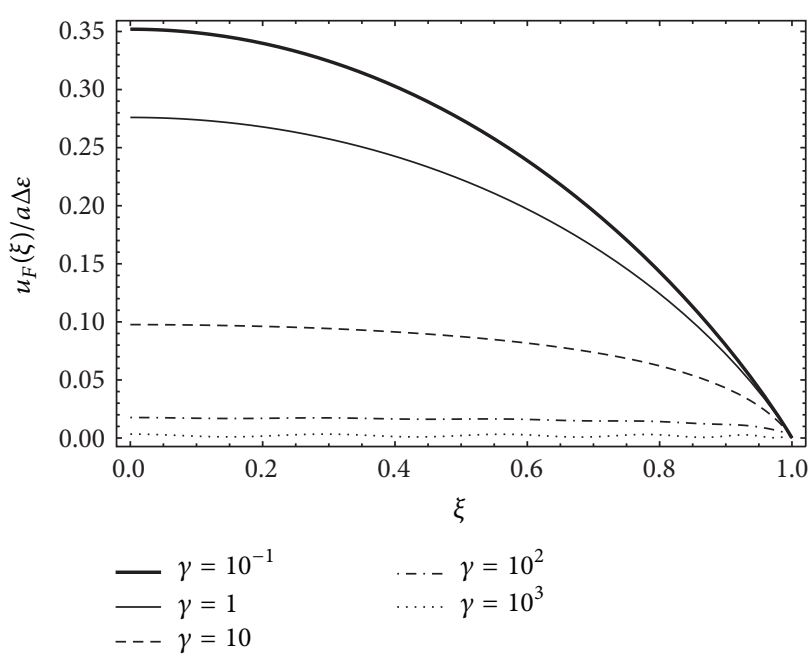

(a)

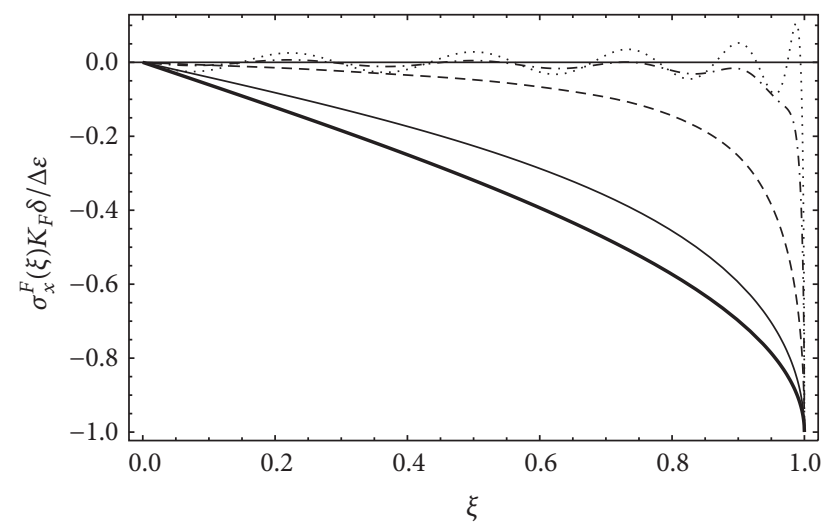

(c)

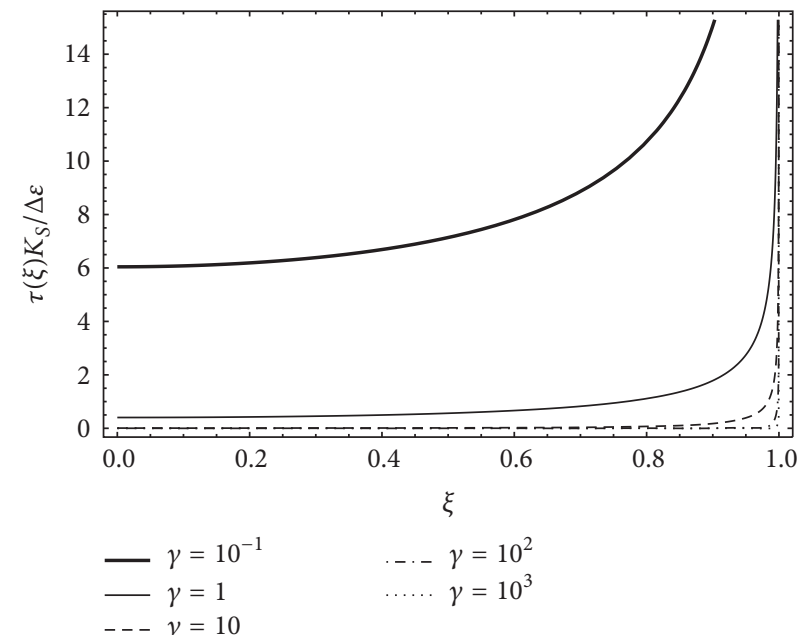

(e)

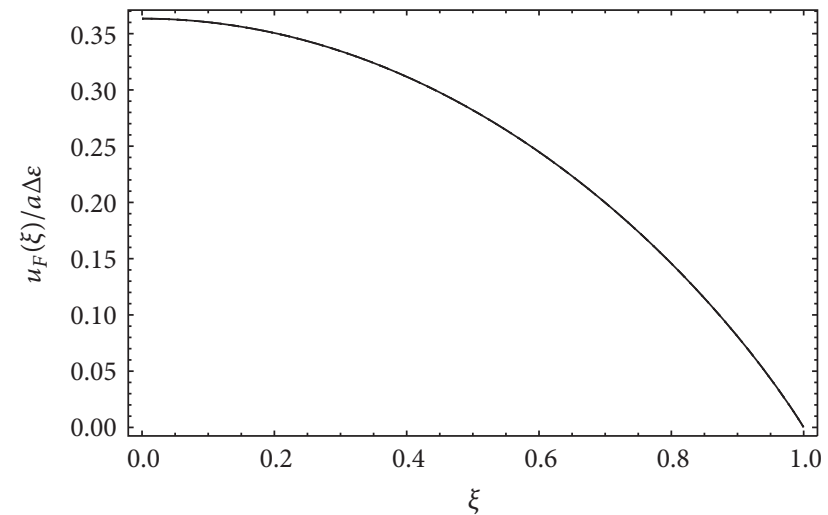

(b)

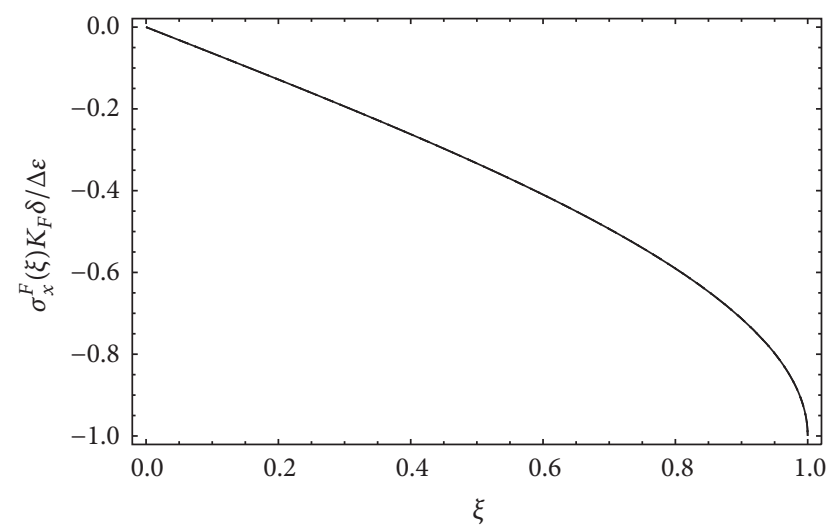

(d)

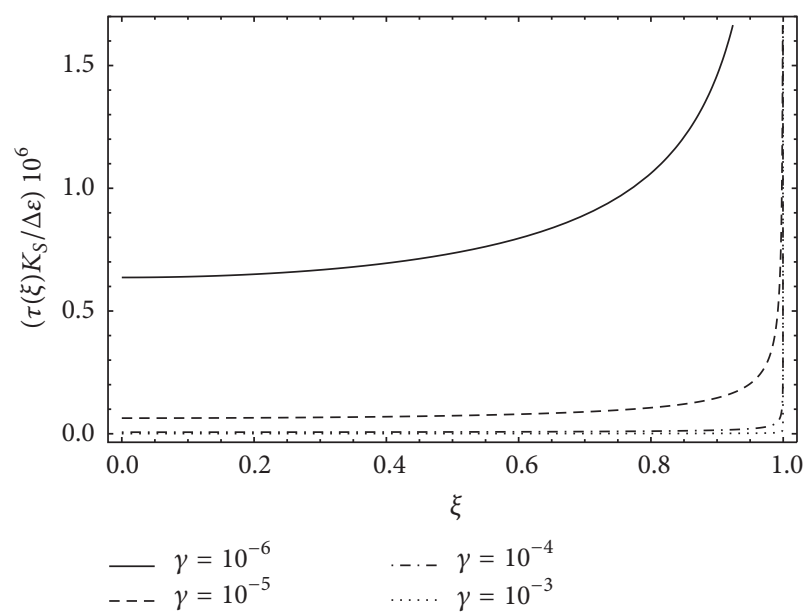

(f)

FIGURE 8: Longitudinal displacement, longitudinal stress, and shear stress field of skew-symmetric loading case condition for high (left (a), (c), and (e)) and low (right (b), (d), and (f)) values of $\gamma$, respectively. 
and, in turn, the film and half plane compliances can be defined as

$$
\begin{aligned}
& K_{F}=\frac{\beta_{22, F}}{\delta}, \\
& K_{S}=\frac{\sqrt{C_{h}+4}}{\lambda} \beta_{22, S} .
\end{aligned}
$$

In case of isotropic half plane, the elastic parameters and compliances reduce to

$$
\begin{aligned}
\beta_{22} & =\frac{1-v^{2}}{E}, \\
\lambda & =1, \\
C_{h} & =0, \\
R & =\frac{(1-2 v)(1+v)}{E}, \\
\tilde{c}_{12} & =E, \\
K_{S} & =\frac{2\left(1-v_{S}^{2}\right)}{E_{S}}, \\
K_{F} & =\frac{1-v_{F}^{2}}{E_{F} \delta},
\end{aligned}
$$

and the half plane longitudinal strain reads [47]

$$
\varepsilon^{S}(y)=\frac{\left(1-v_{S}^{2}\right)}{E \pi} \int_{-a}^{+a} \frac{\tau(t)}{y-t} d t
$$

\section{B. Useful Integral Involving Chebyshev Polynomials}

The Chebyshev polynomials of the first and second kind, $T_{n}(t)$ and $U_{n}(t)$, are defined as

$$
\begin{aligned}
T_{n}(\xi) & =\cos [n \arccos (\xi)], \\
U_{n}(\xi) & =\frac{\sin [(n+1) \arccos (\xi)]}{\sin [\arccos (\xi)]} .
\end{aligned}
$$

In Section 2, the following identities have been used:

$$
\begin{aligned}
& \int_{-a}^{a} \frac{T_{n}(t)}{(t-x) \sqrt{1-t^{2}}} \\
& = \begin{cases}0, & \text { if } n=1,|x|<a \\
\pi U_{n-1}(x), & \text { if } n \neq 1,|x|<a \\
-\pi \frac{\left[x-\operatorname{sign}(x) \sqrt{x^{2}-1}\right]^{n}}{\operatorname{sign}(x) \sqrt{x^{2}-1}}, & \text { if } n \geq 0,|x|>1\end{cases}
\end{aligned}
$$

$$
\begin{aligned}
& \int_{x}^{1} \frac{T_{n}(t)}{\sqrt{1-t^{2}}} d t= \begin{cases}\frac{1}{n} U_{n-1}(x) \sqrt{1-x^{2}}, & \text { if } n \neq 0 \\
\arccos (x), & \text { if } n=0\end{cases} \\
& \int_{-a}^{+a} U_{n-1}(t) U_{m-1}(t) \sqrt{1-t^{2}} d t=\frac{\pi}{2} \delta_{n m}, \\
& \int_{-a}^{a} U_{n-1}(t) d t= \begin{cases}\frac{2}{n}, & \text { if } n \text { odd } \\
0, & \text { if } n \text { even }\end{cases} \\
& \int_{-a}^{+a} \frac{T_{n}(t)}{\sqrt{1-t^{2}}} \ln \left|\frac{x}{a}-t\right| d t= \begin{cases}-\pi \ln (2), & \text { if } n=0 \\
-\frac{\pi}{n} T_{n}(x), & \text { if } n>0 .\end{cases}
\end{aligned}
$$

\section{Competing Interests}

The authors declare that there are no competing interests regarding the publication of this paper.

\section{References}

[1] L. B. Freund and S. Suresh, Thin Film Materials. Stress, Defect Formation and Surface Evolution, Cambridge University Press, 2003.

[2] V. Guidi, L. Lanzoni, and A. Mazzolari, "Patterning and modeling of mechanically bent silicon plates deformed through coactive stresses," Thin Solid Films, vol. 520, no. 3, pp. 1074-1079, 2011.

[3] L. Lanzoni and E. Radi, "Thermally induced deformations in a partially coated elastic layer," International Journal of Solids and Structures, vol. 46, no. 6, pp. 1402-1412, 2009.

[4] V. Guidi, L. Lanzoni, A. Mazzolari, G. Martinelli, and A. Tralli, "Design of a crystalline undulator based on patterning by tensile Si3N4 strips on a Si crystal," Applied Physics Letters, vol. 90, no. 11, Article ID 114107, 2007.

[5] L. Lanzoni, "Analysis of stress singularities in thin coatings bonded to a semi-infinite elastic substrate," International Journal of Solids and Structures, vol. 48, no. 13, pp. 1915-1926, 2011.

[6] M. Ohring, The Materials Science of Thin Films, Academic Press, 1992.

[7] A. Caporale, L. Feo, and R. Luciano, "Damage mechanics of cement concrete modeled as a four-phase composite," Composites Part B: Engineering, vol. 65, pp. 124-130, 2014.

[8] G. Dinelli, G. Belz, C. E. Majorana, and B. A. Schrefler, "Experimental investigation on the use of fly ash for lightweight precast structural elements," Materials and Structures, vol. 29, no. 194, pp. 632-638, 1996.

[9] V. A. Salomoni, C. E. Majorana, G. M. Giannuzzi, and A. Miliozzi, "Thermal-fluid flow within innovative heat storage concrete systems for solar power plants," International Journal of Numerical Methods for Heat and Fluid Flow, vol. 18, no. 7-8, pp. 969-999, 2008.

[10] V. Salomoni, G. Mazzucco, C. Pellegrino, and C. Majorana, "Three-dimensional modelling of bond behaviour between concrete and FRP reinforcement," Engineering Computations, vol. 28, no. 1, pp. 5-29, 2011.

[11] G. Xotta, G. Mazzucco, V. A. Salomoni, C. E. Majorana, and K. J. Willam, "Composite behavior of concrete materials under high 
temperatures," International Journal of Solids and Structures, vol. 64, pp. 86-99, 2015.

[12] A. Nobili, L. Lanzoni, and A. M. Tarantino, "Experimental investigation and monitoring of a polypropylene-based fiber reinforced concrete road pavement," Construction and Building Materials, vol. 47, pp. 888-895, 2013.

[13] L. Dezi, G. Menditto, and A. M. Tarantino, "Homogeneous structures subjected to repeated structural system changes," Journal of Engineering Mechanics, vol. 116, no. 8, pp. 1723-1732, 1990.

[14] L. Dezi and A. Tarantino, "Time dependent analysis of concrete structures with variable structural system," ACI Materials Journal, vol. 88, no. 3, pp. 320-324, 1991.

[15] A. Nobili, "Variational approach to beams resting on twoparameter tensionless elastic foundations," Journal of Applied Mechanics, Transactions ASME, vol. 79, no. 2, Article ID 021010, 2012.

[16] R. Mahajan, F. Erdogan, and Y. T. Chou, "The crack problem for an orthotropic half-plane stiffened by elastic films," International Journal of Engineering Science, vol. 31, no. 3, pp. 403-424, 1993.

[17] R. Mahajan, F. Erdogan, B. Kilic, and E. Madenci, "Cracking of an orthotropic substrate reinforced by an orthotropic plate," International Journal of Solids and Structures, vol. 40, no. 23, pp. 6389-6415, 2003.

[18] A. M. Tarantino, "Nonlinear fracture mechanics for an elastic Bell material," The Quarterly Journal of Mechanics and Applied Mathematics, vol. 50, no. 3, pp. 435-456, 1997.

[19] A. M. Tarantino, "The singular equilibrium field at the notch-tip of a compressible material in finite elastostatics," Zeitschrift für Angewandte Mathematik und Physik, vol. 48, no. 3, pp. 370-388, 1997.

[20] A. M. Tarantino, "On extreme thinning at the notch tip of a neoHookean sheet," The Quarterly Journal of Mechanics and Applied Mathematics, vol. 51, no. 2, pp. 179-190, 1998.

[21] A. M. Tarantino, "On the finite motions generated by a mode I propagating crack," Journal of Elasticity, vol. 57, no. 2, pp. 85103, 1999.

[22] A. M. Tarantino, "Crack propagation in finite elastodynamics," Mathematics and Mechanics of Solids, vol. 10, no. 6, pp. 577-601, 2005.

[23] A. Apuzzo, R. Barretta, and R. Luciano, "Some analytical solutions of functionally graded Kirchhoff plates," Composites Part B: Engineering, vol. 68, pp. 266-269, 2015.

[24] R. Barretta, L. Feo, and R. Luciano, "Some closed-form solutions of functionally graded beams undergoing nonuniform torsion," Composite Structures, vol. 123, pp. 132-136, 2015.

[25] R. Barretta, L. Feo, and R. Luciano, "Torsion of functionally graded nonlocal viscoelastic circular nanobeams," Composites Part B: Engineering, vol. 72, pp. 217-222, 2015.

[26] R. Luciano and J. R. Willis, "Non-local constitutive equations for functionally graded materials," Mechanics of Materials, vol. 36, no. 12, pp. 1195-1206, 2004.

[27] K. N. H. Arutiunian, "Contact problem for half-plane with elastic reinforcement," Journal of Applied Mathematics and Mechanics, vol. 32, no. 4, pp. 632-646, 1968.

[28] F. Erdogan and G. D. Gupta, "Problem of an elastic stiffener bonded to a half plane," Journal of Applied Mechanics, vol. 38, no. 4, pp. 937-941, 1971.

[29] T. W. Shield and K. S. Kim, "Beam theory models for thin film segments cohesively bonded to an elastic half space,"
International Journal of Solids and Structures, vol. 29, no. 9, pp. 1085-1103, 1992.

[30] H. Djabella and R. D. Arnell, "Two-dimensional finite-element analysis of elastic stresses in double-layer systems under combined surface normal and tangential loads," Thin Solid Films, vol. 226, no. 1, pp. 65-73, 1993.

[31] J. F. Luo, Y. J. Liu, and E. J. Berger, "Analysis of two-dimensional thin structures (from micro- to nano-scales) using the boundary element method," Computational Mechanics, vol. 22, no. 5, pp. 404-412, 1998.

[32] N. Tullini, A. Tralli, and L. Lanzoni, "Interfacial shear stress analysis of bar and thin film bonded to 2D elastic substrate using a coupled FE-BIE method," Finite Elements in Analysis and Design, vol. 55, pp. 42-51, 2012.

[33] D. S. Jeng, "Tailoring of silicon crystals for relativistic-particle channeling," Journal of Geotechnical and Geoenvironmental Engineering, vol. 123, 1997.

[34] H. B. Huntington, The Elastic Constants of Crystals, Academic Press, 2003.

[35] EN-Standard, "European non-alloy structural steels," Technical Report, EN 10025-2, 2004.

[36] D. Dinckal, "Analysis of elastic anisotropy of wood material for engineering applications," Journal of Innovative Research in Engineering and Science, vol. 2, no. 2, pp. 67-80, 2011.

[37] O. Tabata, K. Kawahata, S. Sugiyama et al., "Mechanical property meansurements of thin films using load-deection of composite rectangular membrane," in Proceedings of the IEEE An Investigation of Micro Structures, Sensors, Actuators, Machines and Robots, Micro Electro Mechanical Systems, vol. 56, pp. 152156, February 1989.

[38] M. A. Hopcroft, W. D. Nix, and T. W. Kenny, "What is the Young's modulus of Silicon?" Journal of Microelectromechanical Systems, vol. 19, no. 2, Article ID 5430873, pp. 229-238, 2010.

[39] P. Bisegna and R. Luciano, "Bounds on the overall properties of composites with debonded frictionless interfaces," Mechanics of Materials, vol. 28, no. 1-4, pp. 23-32, 1998.

[40] F. Marotti de Sciarra and M. Salerno, "On thermodynamic functions in thermoelasticity without energy dissipation," European Journal of Mechanics. A. Solids, vol. 46, pp. 84-95, 2014.

[41] G. A. Morar and G. I. A. Popov, "On the contact problem for a half-plane with finite elastic reinforcement," Percona Monitoring and Management, vol. 34, no. 3, pp. 412-421, 1970.

[42] W. T. Koiter, "On the diffusion of load from a stiffener into a sheet," The Quarterly Journal of Mechanics and Applied Mathematics, vol. 8, no. 2, pp. 164-178, 1955.

[43] R. Barretta, L. Feo, R. Luciano, and F. Marotti de Sciarra, "A gradient Eringen model for functionally graded nanorods," Composite Structures, vol. 131, pp. 1124-1131, 2015.

[44] R. Barretta, L. Feo, R. Luciano, and F. Marotti de Sciarra, "Variational formulations for functionally graded nonlocal BernoulliEuler nanobeams," Composite Structures, vol. 129, pp. 80-89, 2015.

[45] R. Barretta, R. Luciano, and J. R. Willis, "On torsion of random composite beams," Composite Structures, vol. 132, pp. 915-922, 2015.

[46] R. Luciano and J. R. Willis, "Hashin-Shtrikman based FE analysis of the elastic behaviour of finite random composite bodies," International Journal of Fracture, vol. 137, no. 1-4, pp. 261-273, 2006.

[47] K. L. Johnson, Contact Mechanics, Cambridge University Press, London, UK, 1985. 


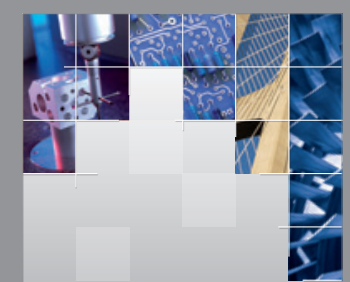

\section{Enfincering}
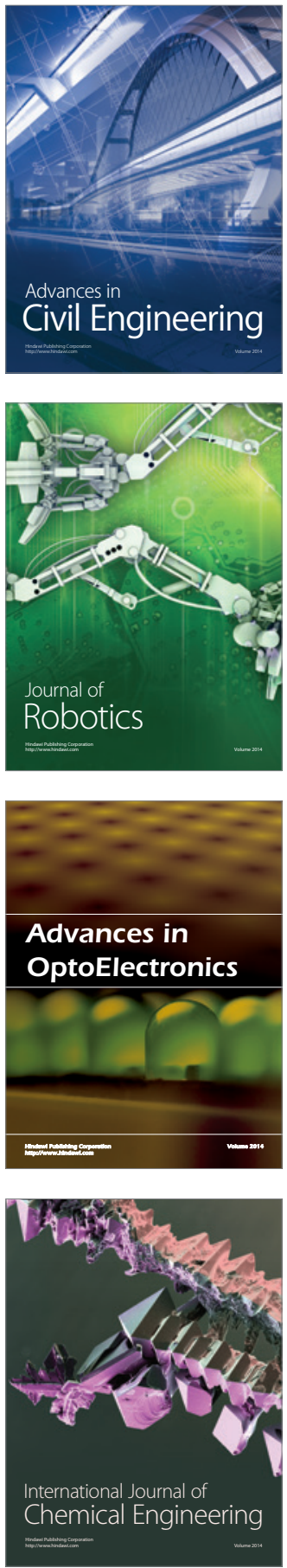

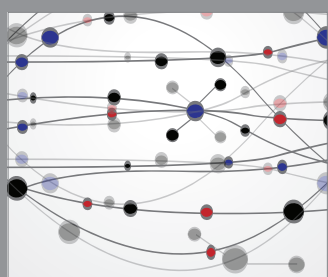

The Scientific World Journal

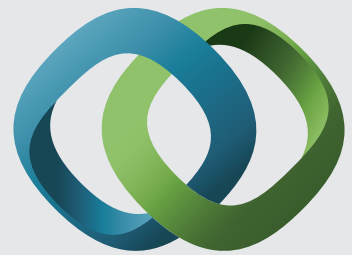

\section{Hindawi}

Submit your manuscripts at

http://www.hindawi.com
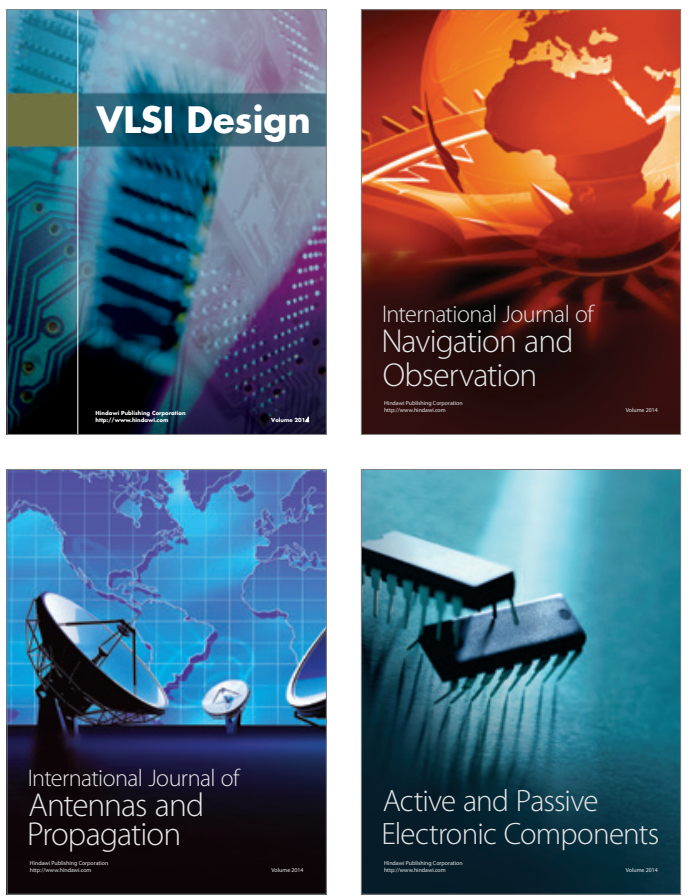
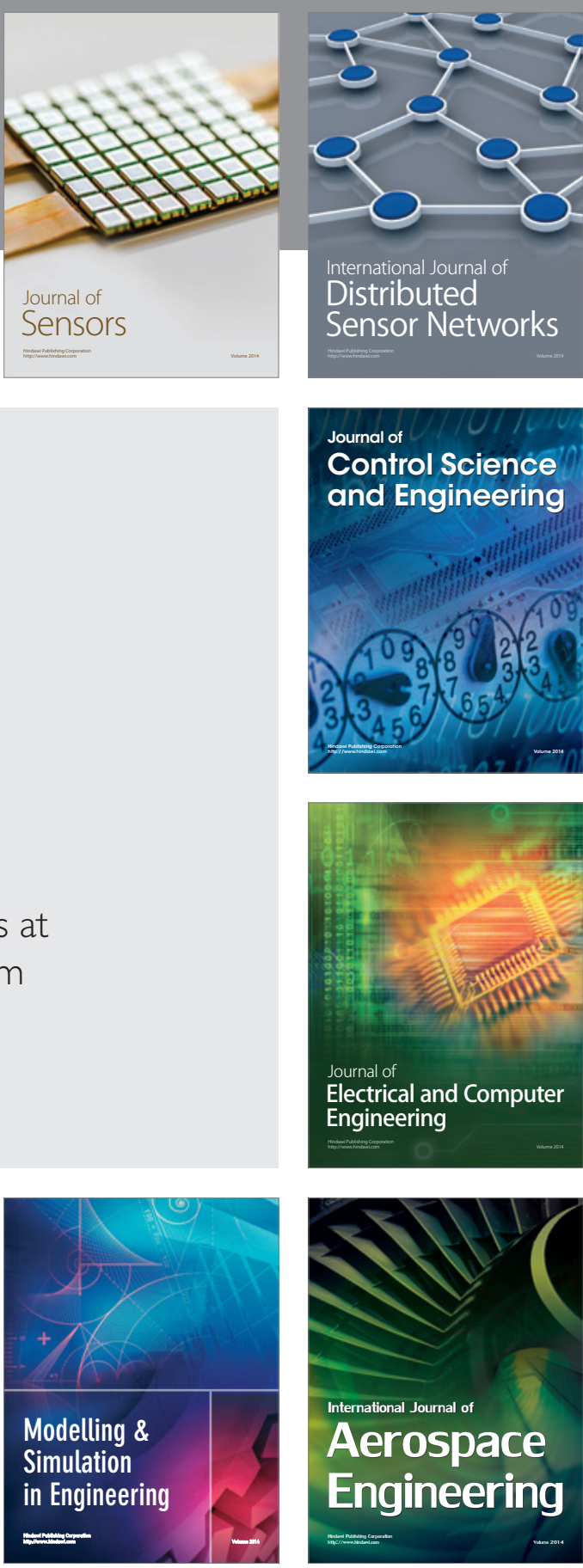

International Journal of

Distributed

Sensor Networks

Journal of

Control Science

and Engineering
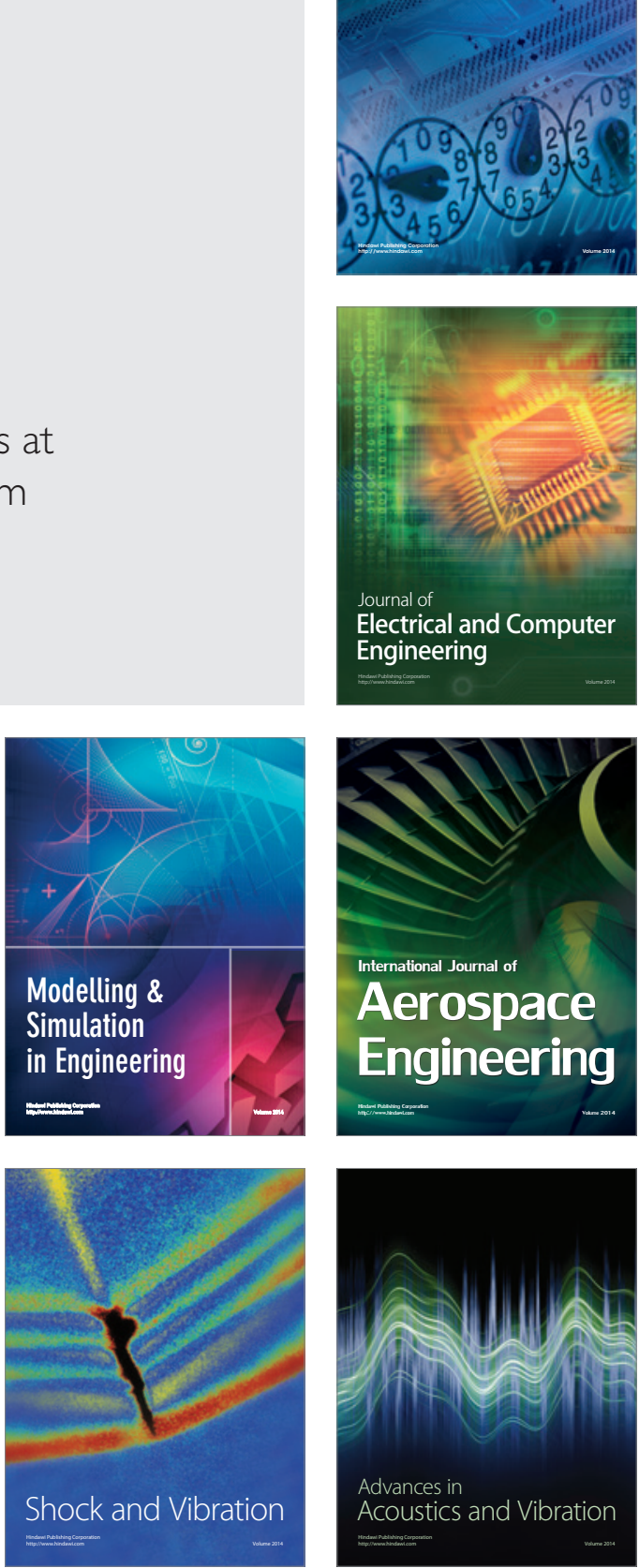